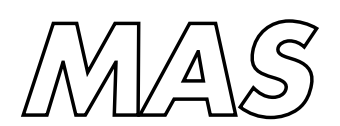

Modelling, Analysis and Simulation

Modelling, Analysis and Simulation

J.L. López, N.M. Temme

REPORT MAS-R021 1 APRIL 30, 2002 
CWI is the National Research Institute for Mathematics and Computer Science. It is sponsored by the Netherlands Organization for Scientific Research (NWO).

$\mathrm{CWI}$ is a founding member of ERCIM, the European Research Consortium for Informatics and Mathematics.

CWI's research has a theme-oriented structure and is grouped into four clusters. Listed below are the names of the clusters and in parentheses their acronyms.

Probability, Networks and Algorithms (PNA)

Software Engineering (SEN)

Modelling, Analysis and Simulation (MAS)

Information Systems (INS)

Copyright @ 2001, Stichting Centrum voor Wiskunde en Informatica

P.O. Box 94079, 1090 GB Amsterdam (NL)

Kruislaan 413, 1098 SJ Amsterdam (NL)

Telephone +31205929333

Telefax +31205924199

ISSN 1386-3703 


\title{
Two-point Taylor Expansions of Analytic Functions
}

\author{
José L. López ${ }^{1}$ and Nico M. Temme ${ }^{2}$ \\ ${ }^{1}$ Departamento de Matématica e Informática, \\ Universidad Pública de Navarra, 31006-Pamplona, Spain \\ ${ }^{2}$ CWI, P.O. Box 94079, 1090 GB Amsterdam, The Netherlands \\ e-mail: jl.lopez@unavarra.es, nicot@cwi.nl
}

\begin{abstract}
Taylor expansions of analytic functions are considered with respect to two points. Cauchy-type formulas are given for coefficients and remainders in the expansions, and the regions of convergence are indicated. It is explained how these expansions can be used in deriving uniform asymptotic expansions of integrals. The method is also used for obtaining Laurent expansions in two points.
\end{abstract}

2000 Mathematics Subject Classification: 30B10, 30E20, 40A30.

Keywords \& Phrases: two-point Taylor expansions, Cauchy's theorem, analytic functions, two-point Laurent expansions, uniform asymptotic expansions of integrals.

Note: Work carried out under project MAS1.2 Analysis, Asymptotics and Computing. This report has been accepted for publication in Studies in Applied Mathematics.

\section{Introduction}

In deriving uniform asymptotic expansions of a certain class of integrals one encounters the problem of expanding a function, that is analytic in some domain $\Omega$ of the complex plane, in two points. The first mention of the use of such expansions in asymptotics is given in [1], where Airy-type expansions are derived for integrals having two nearby (or coalescing) saddle points. This reference does not give further details about two-point Taylor expansions, because the coefficients in the Airy-type asymptotic expansion are derived in a different way.

To demonstrate the application in asymptotics we consider the integral

$$
F_{b}(\omega)=\frac{1}{2 \pi i} \int_{\mathcal{C}} e^{\omega\left(\frac{1}{3} z^{3}-b^{2} z\right)} f(z) d z
$$

where $\omega$ is a large positive parameter and $b$ is a parameter that may assume small values. The contour starts at $\infty e^{-i \pi / 3}$ and terminates at $\infty e^{i \pi / 3}$, and lies in a domain 
where the function $f$ is analytic. In particular, $f$ is analytic in a domain that contains the saddle points $\pm b$ of the exponent in the integrand. One method for obtaining an asymptotic expansion of $F_{b}(\omega)$ that holds uniformly for small values of $b$ is based on expanding $f$ at the two saddle points:

$$
f(z)=\sum_{n=0}^{\infty} A_{n}\left(z^{2}-b^{2}\right)^{n}+z \sum_{n=0}^{\infty} B_{n}\left(z^{2}-b^{2}\right)^{n}
$$

and substitute this expansion into (1). When interchanging summation and integration, the result is a formal expansion in two series in terms of functions related with Airy functions. A Maple algorithm for obtaining the coefficients $A_{n}$ and $B_{n}$, with applications to Airy-type expansions of parabolic cylinder functions, is given in [4].

In a future paper we use expansions like (2) in order to derive convergent expansions for orthogonal polynomials and hypergeometric functions that also have an asymptotic nature. The purpose of the present paper is to give details on the two-point Taylor expansion (2), in particular on the region of convergence and on representations in terms of Cauchy-type integrals of coefficients and remainders of these expansions. Some information on this type of expansions is also given in [6], p. 149, Exercise 24.

Without referring to applications in asymptotic analysis we include analogous properties of two-point Laurent expansions and of another related type, the two-point TaylorLaurent expansion.

\section{Two-point Taylor expansions}

We consider the expansion (2) in a more symmetric form and give information on the coefficients and the remainder in the expansion.

Theorem 1. Let $f(z)$ be an analytic function on an open set $\Omega \subset \mathbb{C}$ and $z_{1}, z_{2} \in \Omega$ with $z_{1} \neq z_{2}$. Then, $f(z)$ admits the two-point Taylor expansion

$$
f(z)=\sum_{n=0}^{N-1}\left[a_{n}\left(z_{1}, z_{2}\right)\left(z-z_{1}\right)+a_{n}\left(z_{2}, z_{1}\right)\left(z-z_{2}\right)\right]\left(z-z_{1}\right)^{n}\left(z-z_{2}\right)^{n}+r_{N}\left(z_{1}, z_{2} ; z\right),
$$

where the coefficients $a_{n}\left(z_{1}, z_{2}\right)$ and $a_{n}\left(z_{2}, z_{1}\right)$ of the expansion are given by the Cauchy integral

$$
a_{n}\left(z_{1}, z_{2}\right) \equiv \frac{1}{2 \pi i\left(z_{2}-z_{1}\right)} \int_{\mathcal{C}} \frac{f(w) d w}{\left(w-z_{1}\right)^{n}\left(w-z_{2}\right)^{n+1}} .
$$

The remainder term $r_{N}\left(z_{1}, z_{2} ; z\right)$ is given by the Cauchy integral

$$
r_{N}\left(z_{1}, z_{2} ; z\right) \equiv \frac{1}{2 \pi i} \int_{\mathcal{C}} \frac{f(w) d w}{\left(w-z_{1}\right)^{N}\left(w-z_{2}\right)^{N}(w-z)}\left(z-z_{1}\right)^{N}\left(z-z_{2}\right)^{N} .
$$

The contour of integration $\mathcal{C}$ is a simple closed loop which encircles the points $z_{1}$ and $z_{2}\left(\right.$ for $\left.a_{n}\right)$ and $z, z_{1}$ and $z_{2}\left(\right.$ for $\left.r_{N}\right)$ in the counterclockwise direction and is contained in $\Omega$ (see Figure $1(a)$ ). 
The expansion (3) is convergent for $z$ inside the Cassini oval (see Figure 2)

$$
O_{z_{1}, z_{2}} \equiv\left\{z \in \Omega,\left|\left(z-z_{1}\right)\left(z-z_{2}\right)\right|<r\right\}
$$

where

$$
r \equiv \operatorname{Inf}_{w \in \mathbb{C} \backslash \Omega}\left\{\left|\left(w-z_{1}\right)\left(w-z_{2}\right)\right|\right\} .
$$

In particular, if $f(z)$ is an entire function $(\Omega=\mathbb{C})$, then the expansion (3) converges $\forall$ $z \in \mathbb{C}$.

Proof. By Cauchy's theorem,

$$
f(z)=\frac{1}{2 \pi i} \int_{\mathcal{C}} \frac{f(w) d w}{w-z}
$$

where $\mathcal{C}$ is the contour defined above (Figure $1(\mathrm{a})$ ). Now we write

$$
\frac{1}{w-z}=\frac{z+w-z_{1}-z_{2}}{\left(w-z_{1}\right)\left(w-z_{2}\right)} \frac{1}{1-u}
$$

where

$$
u \equiv \frac{\left(z-z_{1}\right)\left(z-z_{2}\right)}{\left(w-z_{1}\right)\left(w-z_{2}\right)} .
$$

Now we introduce the expansion

$$
\frac{1}{1-u}=\sum_{n=0}^{N-1} u^{n}+\frac{u^{N}}{1-u}
$$

in (7) and this in (6). After straightforward calculations we obtain (3)-(5).

For any $z \in O_{z_{1}, z_{2}}$, we can take a contour $\mathcal{C}$ in $\Omega$ such that $\left|\left(z-z_{1}\right)\left(z-z_{2}\right)\right|<$ $\left|\left(w-z_{1}\right)\left(w-z_{2}\right)\right| \forall w \in \mathcal{C}$ (see Figure $\left.1(\mathrm{~b})\right)$. In this contour $|f(w)|$ is bounded by some constant $C:|f(w)| \leq C$. Introducing these two bounds in (5) we see that $\lim _{N \rightarrow \infty} r_{N}\left(z_{1}, z_{2} ; z\right)=0$ and the proof follows.

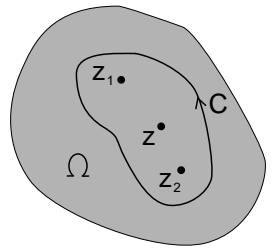

(a)

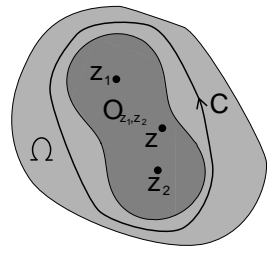

(b)

Figure 1. (a) Contour $\mathcal{C}$ in the integrals (3)-(5). (b) For $z \in O_{z_{1}, z_{2}}$, we can take a contour $\mathcal{C}$ in $\Omega$ which contains $O_{z_{1}, z_{2}}$ inside and therefore, $\left|\left(z-z_{1}\right)\left(z-z_{2}\right)\right|<\left|\left(w-z_{1}\right)\left(w-z_{2}\right)\right| \forall$ $w \in \mathcal{C}$. 


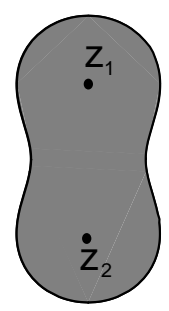

(a) $4 r>\left|z_{1}-z_{2}\right|^{2}$

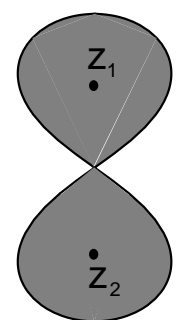

(b) $4 r=\left|z_{1}-z_{2}\right|^{2}$
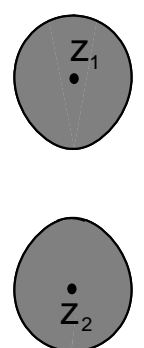

(c) $4 r<\left|z_{1}-z_{2}\right|^{2}$

Figure 2. Shape of the Cassini oval depending on the relative size of the parameter $r$ and the focal distance $\left|z_{1}-z_{2}\right|$.

\subsection{An alternative form of the expansion}

The present expansion of $f(z)$ in the form (3) stresses the symmetry of the expansion with respect to $z_{1}$ and $z_{2}$. In this representation it is not possible, however, to let $z_{1}$ and $z_{2}$ coincide, which causes a little inconvenience (the coefficients $a_{n}\left(z_{1}, z_{2}\right)$ become infinitely large as $z_{1} \rightarrow z_{2}$; the remainder $r_{N}\left(z_{1}, z_{2} ; z\right)$ remains well-defined). An alternative way is the representation (cf. (2)),

$$
f(z)=\sum_{n=0}^{\infty}\left[A_{n}\left(z_{1}, z_{2}\right)+B_{n}\left(z_{1}, z_{2}\right) z\right]\left(z-z_{1}\right)^{n}\left(z-z_{2}\right)^{n},
$$

and we have the relations

$$
\begin{aligned}
& A_{n}\left(z_{1}, z_{2}\right)=-z_{1} a_{n}\left(z_{1}, z_{2}\right)-z_{2} a_{n}\left(z_{2}, z_{1}\right), \\
& B_{n}\left(z_{1}, z_{2}\right)=a_{n}\left(z_{1}, z_{2}\right)+a_{n}\left(z_{2}, z_{1}\right),
\end{aligned}
$$

which are regular when $z_{1} \rightarrow z_{2}$. In fact we have

$$
\begin{aligned}
& A_{n}\left(z_{1}, z_{2}\right)=\frac{1}{2 \pi i} \int_{\mathcal{C}} \frac{w-z_{1}-z_{2}}{\left[\left(w-z_{1}\right)\left(w-z_{2}\right)\right]^{n+1}} f(w) d w \\
& B_{n}\left(z_{1}, z_{2}\right)=\frac{1}{2 \pi i} \int_{\mathcal{C}} \frac{f(w) d w}{\left[\left(w-z_{1}\right)\left(w-z_{2}\right)\right]^{n+1}} .
\end{aligned}
$$

Letting $z_{1} \rightarrow 0$ and $z_{2} \rightarrow 0$, we obtain the standard Maclaurin series of $f(z)$ with even part (the $A_{n}$ series) and odd part (the $B_{n}$ series).

\subsection{Explicit forms of the coefficients}

Definition (4) is not appropriate for numerical computations. A more practical formula to compute the coefficients of the above two-point Taylor expansion is given in the following proposition.

Proposition 1. Coefficients $a_{n}\left(z_{1}, z_{2}\right)$ in the expansion (3) are also given by the formulas:

$$
a_{0}\left(z_{1}, z_{2}\right)=\frac{f\left(z_{2}\right)}{z_{2}-z_{1}}
$$


and, for $n=1,2,3, \ldots$,

$$
a_{n}\left(z_{1}, z_{2}\right)=\sum_{k=0}^{n} \frac{(n+k-1) !}{k !(n-k) !} \frac{(-1)^{n+1} n f^{(n-k)}\left(z_{2}\right)+(-1)^{k} k f^{(n-k)}\left(z_{1}\right)}{n !\left(z_{1}-z_{2}\right)^{n+k+1}} .
$$

Proof. We deform the contour of integration $\mathcal{C}$ in equation (4) to any contour of the form $\mathcal{C}_{1} \cup \mathcal{C}_{2}$ also contained in $\Omega$, where $\mathcal{C}_{1}\left(\mathcal{C}_{2}\right)$ is a simple closed loop which encircles the point $z_{1}\left(z_{2}\right)$ in the counterclockwise direction and does not contain the point $z_{2}\left(z_{1}\right)$ inside (see Figure $3($ a)). Then,

$$
\begin{aligned}
a_{n}\left(z_{1}, z_{2}\right)= & \frac{1}{2 \pi i\left(z_{2}-z_{1}\right)}\left\{\int_{\mathcal{C}_{1}} \frac{f(w)}{\left(w-z_{2}\right)^{n+1}} \frac{d w}{\left(w-z_{1}\right)^{n}}+\int_{\mathcal{C}_{2}} \frac{f(w)}{\left(w-z_{1}\right)^{n}} \frac{d w}{\left(w-z_{2}\right)^{n+1}}\right\}= \\
& \frac{1}{\left(z_{2}-z_{1}\right)}\left\{\left.\frac{1}{(n-1) !} \frac{d^{n-1}}{d w^{n-1}} \frac{f(w)}{\left(w-z_{2}\right)^{n+1}}\right|_{w=z_{1}}+\left.\frac{1}{n !} \frac{d^{n}}{d w^{n}} \frac{f(w)}{\left(w-z_{1}\right)^{n}}\right|_{w=z_{2}}\right\} .
\end{aligned}
$$

From here, equations (10)-(11) follows after straightforward computations.

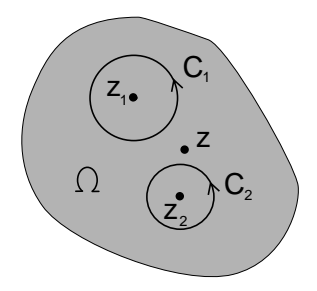

(a)

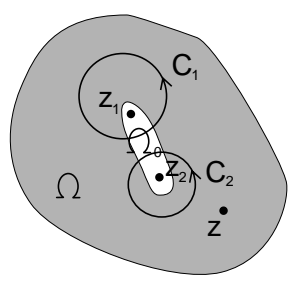

(b)

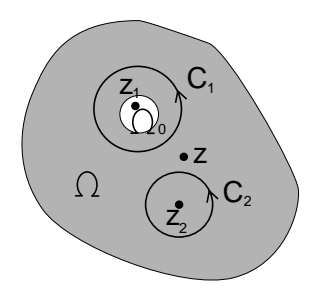

(c)

Figure 3. (a) The function $\left(w-z_{2}\right)^{-n-1} f(w)$ is analytic inside $\mathcal{C}_{1}$, whereas $\left(w-z_{1}\right)^{-n} f(w)$ is analytic inside $\mathcal{C}_{2}$. (b) The function $\left(w-z_{2}\right)^{-n-1} g_{1}(w)$ is analytic inside $\mathcal{C}_{1}$, whereas $(w-$ $\left.z_{1}\right)^{-n} g_{2}(w)$ is analytic inside $\mathcal{C}_{2}$. (c) The function $\left(w-z_{2}\right)^{-n-1} g(w)$ is analytic inside $\mathcal{C}_{1}$, whereas $\left(w-z_{1}\right)^{-n} f(w)$ is analytic inside $\mathcal{C}_{2}$.

\subsection{Two-point Taylor polynomials}

Next we can define the two-point Taylor polynomial of the function $f(z)$ at in the following way:

Definition 1. Let $z$ be a real or complex variable and $z_{1}$ and $z_{2}\left(z_{1} \neq z_{2}\right)$ two real or complex numbers. If $f(z)$ is $n-1$-times differentiable at those two points, we define the two-point Taylor polynomial of $f(z)$ at $z_{1}$ and $z_{2}$ and degree $2 n-1$ as

$$
P_{n}\left(z_{1}, z_{2} ; z\right) \equiv \sum_{k=0}^{n-1}\left[a_{k}\left(z_{1}, z_{2}\right)\left(z-z_{1}\right)+a_{k}\left(z_{2}, z_{1}\right)\left(z-z_{2}\right)\right]\left(z-z_{1}\right)^{k}\left(z-z_{2}\right)^{k}
$$

where the coefficients $a_{k}\left(z_{1}, z_{2}\right)$ are given in (10)-(11). 
Proposition 2. In the conditions of the above definition, define the remainder of the approximation of $f(z)$ by $P_{n}\left(z_{1}, z_{2} ; z\right)$ at $z_{1}$ and $z_{2}$ as

$$
r_{n}\left(z_{1}, z_{2} ; z\right) \equiv f(z)-P_{n}\left(z_{1}, z_{2} ; z\right) .
$$

Then, (i) $r_{n}\left(z_{1}, z_{2} ; z\right)=o\left(z-z_{1}\right)^{n-1}$ as $z \rightarrow z_{1}$ and $r_{n}\left(z_{1}, z_{2} ; z\right)=o\left(z-z_{2}\right)^{n-1}$ as $z \rightarrow$ $z_{2}$. (ii) If $f(z)$ is n-times differentiable at $z_{1}$ and/or $z_{2}$, then $r_{n}\left(z_{1}, z_{2} ; z\right)=\mathcal{O}\left(z-z_{1}\right)^{n}$ as $z \rightarrow z_{1}$ and/or $r_{n}\left(z_{1}, z_{2} ; z\right)=\mathcal{O}\left(z-z_{2}\right)^{n}$ as $z \rightarrow z_{2}$.

Proof. The proof is trivial if $f(z)$ is analytic at $z_{1}$ and $z_{2}$ by using (5). In any case, for real or complex variable, the proof follows after straightforward computations by using l'Hôpital's rule and (10)-(11).

Remark 1. Observe that the Taylor polynomial of $f(z)$ at $z_{1}$ and $z_{2}$ and degree $2 n-1$ is the same as the Hermite's interpolation polynomial of $f(z)$ at $z_{1}$ and $z_{2}$ with data $f\left(z_{i}\right), f^{\prime}\left(z_{i}\right), \ldots, f^{(n-1)}\left(z_{i}\right), i=1,2$.

\section{Two-point Laurent expansions}

In the standard theory for Taylor and Laurent expansions much analogy exists between the two types of expansions. For two-point expansions, we have a similar agreement in the representations of coefficients and remainders.

Theorem 2. Let $\Omega_{0}$ and $\Omega$ be closed and open sets, respectively, of the complex plane, and $\Omega_{0} \subset \Omega \subset \mathbb{C}$. Let $f(z)$ be an analytic function on $\Omega \backslash \Omega_{0}$ and $z_{1}, z_{2} \in \Omega_{0}$ with $z_{1} \neq z_{2}$. Then, for any $z \in \Omega \backslash \Omega_{0}, f(z)$ admits the two-point Laurent expansion

$$
\begin{aligned}
f(z)= & \sum_{n=0}^{N-1}\left[b_{n}\left(z_{1}, z_{2}\right)\left(z-z_{1}\right)+b_{n}\left(z_{2}, z_{1}\right)\left(z-z_{2}\right)\right]\left(z-z_{1}\right)^{n}\left(z-z_{2}\right)^{n}+ \\
& \sum_{n=0}^{N-1}\left[c_{n}\left(z_{1}, z_{2}\right)\left(z-z_{1}\right)+c_{n}\left(z_{2}, z_{1}\right)\left(z-z_{2}\right)\right]\left(z-z_{1}\right)^{-n-1}\left(z-z_{2}\right)^{-n-1}+ \\
& r_{N}\left(z_{1}, z_{2} ; z\right),
\end{aligned}
$$

where the coefficients $b_{n}\left(z_{1}, z_{2}\right), b_{n}\left(z_{2}, z_{1}\right), c_{n}\left(z_{1}, z_{2}\right)$ and $c_{n}\left(z_{2}, z_{1}\right)$ of the expansion are given, respectively, by the Cauchy integrals

$$
b_{n}\left(z_{1}, z_{2}\right) \equiv \frac{1}{2 \pi i\left(z_{2}-z_{1}\right)} \int_{\Gamma_{1}} \frac{f(w) d w}{\left(w-z_{1}\right)^{n}\left(w-z_{2}\right)^{n+1}}
$$

and

$$
c_{n}\left(z_{1}, z_{2}\right) \equiv \frac{1}{2 \pi i\left(z_{2}-z_{1}\right)} \int_{\Gamma_{2}}\left(w-z_{1}\right)^{n+1}\left(w-z_{2}\right)^{n} f(w) d w .
$$

The remainder term $r_{N}\left(z_{1}, z_{2} ; z\right)$ is given by the Cauchy integrals

$$
\begin{aligned}
r_{N}\left(z_{1}, z_{2} ; z\right) \equiv & \frac{1}{2 \pi i} \int_{\Gamma_{1}} \frac{f(w) d w}{\left(w-z_{1}\right)^{N}\left(w-z_{2}\right)^{N}(w-z)}\left(z-z_{1}\right)^{N}\left(z-z_{2}\right)^{N}- \\
& \frac{1}{2 \pi i} \int_{\Gamma_{2}} \frac{\left(w-z_{1}\right)^{N}\left(w-z_{2}\right)^{N} f(w) d w}{w-z} \frac{1}{\left(z-z_{1}\right)^{N}\left(z-z_{2}\right)^{N}} .
\end{aligned}
$$


In these integrals, the contours of integration $\Gamma_{1}$ and $\Gamma_{2}$ are simple closed loops contained in $\Omega \backslash \Omega_{0}$ which encircle the points $z_{1}$ and $z_{2}$ in the counterclockwise direction. Moreover, $\Gamma_{2}$ does not contain the point $z$ inside, whereas $\Gamma_{1}$ encircles $\Gamma_{2}$ and the point $z$ (see Figure 4 (a)).

The expansion (12) is convergent for $z$ inside the Cassini annulus (see Figure 5)

$$
A_{z_{1}, z_{2}} \equiv\left\{z \in \Omega \backslash \Omega_{0}, \quad r_{2}<\left|\left(z-z_{1}\right)\left(z-z_{2}\right)\right|<r_{1}\right\}
$$

where

$$
r_{1} \equiv \operatorname{Inf}_{w \in \mathbb{C} \backslash \Omega}\left\{\left|\left(w-z_{1}\right)\left(w-z_{2}\right)\right|\right\}, \quad r_{2} \equiv \operatorname{Sup}_{w \in \Omega_{0}}\left\{\left|\left(w-z_{1}\right)\left(w-z_{2}\right)\right|\right\} .
$$

Proof. By Cauchy's theorem,

$$
f(z)=\frac{1}{2 \pi i} \int_{\Gamma_{1}} \frac{f(w) d w}{w-z}-\frac{1}{2 \pi i} \int_{\Gamma_{2}} \frac{f(w) d w}{w-z},
$$

where $\Gamma_{1}$ and $\Gamma_{2}$ are the contours defined above. We substitute (7)-(8) into the first integral above and

$$
\frac{1}{w-z}=\frac{z_{1}+z_{2}-z-w}{\left(z-z_{1}\right)\left(z-z_{2}\right)} \frac{1}{1-u}, \quad u \equiv \frac{\left(w-z_{1}\right)\left(w-z_{2}\right)}{\left(z-z_{1}\right)\left(z-z_{2}\right)}
$$

into the second one. Now we introduce the expansion (9) of the factor $(1-u)^{-1}$ in both integrals in (17). After straightforward calculations we obtain (12)-(15).

For any $z$ verifying (16), we can take simple closed loops $\Gamma_{1}$ and $\Gamma_{2}$ in $\Omega \backslash \Omega_{0}$ such that $\left|\left(z-z_{1}\right)\left(z-z_{2}\right)\right|<\left|\left(w-z_{1}\right)\left(w-z_{2}\right)\right| \forall w \in \Gamma_{1}$ and $\left|\left(z-z_{1}\right)\left(z-z_{2}\right)\right|>\left|\left(w-z_{1}\right)\left(w-z_{2}\right)\right|$ $\forall w \in \Gamma_{2}$ (see Figure $4(\mathrm{~b})$ ). On these contours $|f(w)|$ is bounded by some constant $C:|f(w)| \leq C$. Introducing these bounds in (15) we see that $\lim _{N \rightarrow \infty} r_{N}\left(z_{1}, z_{2} ; z\right)=0$ and the proof follows.

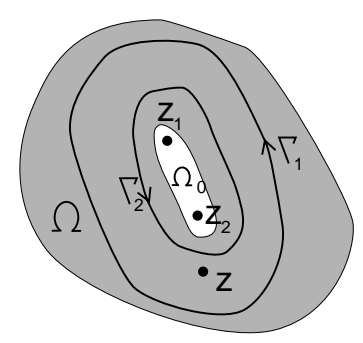

(a)

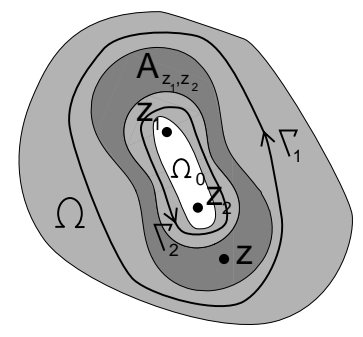

(b)

Figure 4. (a) Contours $\Gamma_{1}$ and $\Gamma_{2}$ in the integrals (12)-(15). (b) For $z \in A_{z_{1}, z_{2}}$, we can take a contour $\Gamma_{2}$ in $\Omega$ situated between $\Omega_{0}$ and $A_{z_{1}, z_{2}}$ and a contour $\Gamma_{1}$ in $\Omega$ which contains $A_{z_{1}, z_{2}}$ inside. Therefore, $\left|\left(z-z_{1}\right)\left(z-z_{2}\right)\right|<\left|\left(w-z_{1}\right)\left(w-z_{2}\right)\right| \forall w \in \Gamma_{1}$ and $\left|\left(w-z_{1}\right)\left(w-z_{2}\right)\right|<$ $\left|\left(z-z_{1}\right)\left(z-z_{2}\right)\right| \forall w \in \Gamma_{2}$. 

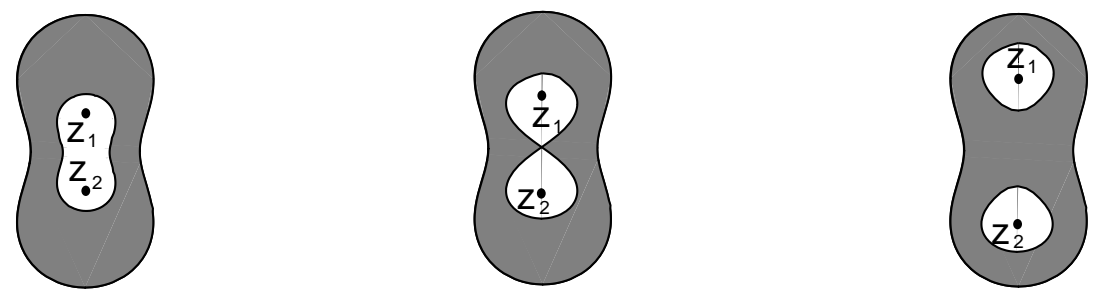

(a) $4 r_{1}>4 r_{2}>\left|z_{1}-z_{2}\right|^{2}$

(b) $4 r_{1}>\left|z_{1}-z_{2}\right|^{2}=4 r_{2}$

(c) $4 r_{1}>\left|z_{1}-z_{2}\right|^{2}>4 r_{2}$
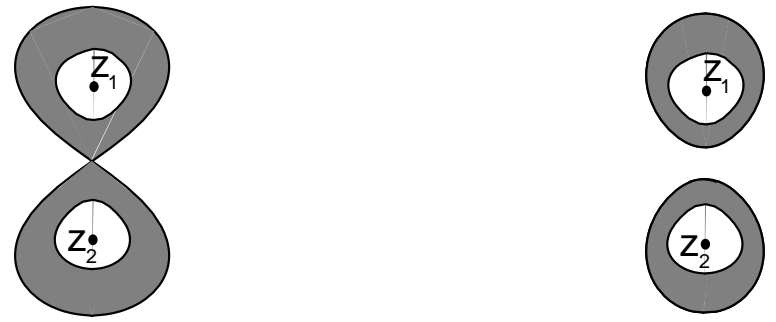

(d) $4 r_{1}=\left|z_{1}-z_{2}\right|^{2}>4 r_{2}$

(e) $\left|z_{1}-z_{2}\right|^{2}>4 r_{1}>4 r_{2}$

Figure 5. Shape of the Cassini annulus depending on the relative size of the parameters $r_{1}, r_{2}$ and the focal distance $\left|z_{1}-z_{2}\right|$.

If the only singularities of $f(z)$ inside $\Omega_{0}$ are just poles at $z_{1}$ and/or $z_{2}$, then an alternative formula to (13) and (14) to compute the coefficients of the above two-point Laurent expansion is given in the following proposition.

Proposition 3. Suppose that $g_{1}(z) \equiv\left(z-z_{1}\right)^{m_{1}} f(z)$ and $g_{2}(z) \equiv\left(z-z_{2}\right)^{m_{2}} f(z)$ are analytic functions in $\Omega$ for certain $m_{1}, m_{2} \in \mathbb{N}$. Then, for $n=0,1,2, \ldots$, coefficients $b_{n}\left(z_{1}, z_{2}\right)$ and $c_{n}\left(z_{1}, z_{2}\right)$ in the expansion (12) are also given by the formulas:

$$
\begin{aligned}
b_{n}\left(z_{1}, z_{2}\right)= & \sum_{k=0}^{n+m_{1}-1}\left(\begin{array}{c}
n+m_{1}-1 \\
k
\end{array}\right) \frac{(-1)^{k+1}(n+1)_{k} g_{1}^{\left(n+m_{1}-k-1\right)}\left(z_{1}\right)}{\left(n+m_{1}-1\right) !\left(z_{1}-z_{2}\right)^{n+k+2}}+ \\
& \sum_{k=0}^{n+m_{2}}\left(\begin{array}{c}
n+m_{2} \\
k
\end{array}\right) \frac{(-1)^{k}(n)_{k} g_{2}^{\left(n+m_{2}-k\right)}\left(z_{2}\right)}{\left(n+m_{2}\right) !\left(z_{2}-z_{1}\right)^{n+k+1}}
\end{aligned}
$$

where $(n)_{k}$ denotes the Pochhammer symbol and

$$
\begin{aligned}
c_{n}\left(z_{1}, z_{2}\right)= & -\sum_{k=0}^{m_{1}-n-2} k !\left(\begin{array}{c}
m_{1}-n-2 \\
k
\end{array}\right)\left(\begin{array}{c}
n \\
k
\end{array}\right) \frac{\left(z_{1}-z_{2}\right)^{n-k-1} g_{1}^{\left(m_{1}-n-k-2\right)}\left(z_{1}\right)}{\left(m_{1}-n-2\right) !}+ \\
& \sum_{k=0}^{m_{2}-n-1} k !\left(\begin{array}{c}
m_{2}-n-1 \\
k
\end{array}\right)\left(\begin{array}{c}
n+1 \\
k
\end{array}\right) \frac{\left(z_{2}-z_{1}\right)^{n-k} g_{2}^{\left(m_{2}-n-k-1\right)}\left(z_{2}\right)}{\left(m_{2}-n-1\right) !} .
\end{aligned}
$$

In these formulas, empty sums must be understood as zero. Coefficients $b_{n}\left(z_{2}, z_{1}\right)$ and $c_{n}\left(z_{2}, z_{1}\right)$ are given, respectively, by (18) and (19) interchanging $z_{1}, g_{1}$ and $m_{1}$ by $z_{2}$, $g_{2}$ and $m_{2}$ respectively. 
Proof. We deform both, the contour $\Gamma_{1}$ in equation (13) and $\Gamma_{2}$ in equation (14), to any contour of the form $\mathcal{C}_{1} \cup \mathcal{C}_{2}$ contained in $\Omega$, where $\mathcal{C}_{1}\left(\mathcal{C}_{2}\right)$ is a simple closed loop which encircles the point $z_{1}\left(z_{2}\right)$ in the counterclockwise direction and does not contain the point $z_{2}\left(z_{1}\right)$ inside (see Figure $\left.3(\mathrm{~b})\right)$. Then,

$$
\begin{aligned}
b_{n}\left(z_{1}, z_{2}\right)= & \frac{1}{2 \pi i\left(z_{2}-z_{1}\right)}\left\{\int_{\mathcal{C}_{1}} \frac{g_{1}(w)}{\left(w-z_{2}\right)^{n+1}} \frac{d w}{\left(w-z_{1}\right)^{n+m_{1}}}+\right. \\
& \left.\int_{\mathcal{C}_{2}} \frac{g_{2}(w)}{\left(w-z_{1}\right)^{n}} \frac{d w}{\left(w-z_{2}\right)^{n+m_{2}+1}}\right\}= \\
& \frac{1}{z_{2}-z_{1}}\left\{\left.\frac{1}{\left(n+m_{1}-1\right) !} \frac{d^{n+m_{1}-1}}{d w^{n+m_{1}-1}} \frac{g_{1}(w)}{\left(w-z_{2}\right)^{n+1}}\right|_{w=z_{1}}+\right. \\
& \left.\left.\frac{1}{\left(n+m_{2}\right) !} \frac{d^{n+m_{2}}}{d w^{n+m_{2}}} \frac{g_{2}(w)}{\left(w-z_{1}\right)^{n}}\right|_{w=z_{2}}\right\}
\end{aligned}
$$

and

$$
\begin{aligned}
& c_{n}\left(z_{1}, z_{2}\right)=\frac{1}{2 \pi i\left(z_{2}-z_{1}\right)}\left\{\int_{\mathcal{C}_{1}} \frac{\left(w-z_{2}\right)^{n} g_{1}(w)}{\left(w-z_{1}\right)^{m_{1}-n-1}} d w+\int_{\mathcal{C}_{2}} \frac{\left(w-z_{1}\right)^{n+1} g_{2}(w)}{\left(w-z_{2}\right)^{m_{2}-n}} d w\right\}= \\
& \frac{1}{z_{2}-z_{1}}\left\{\left.\frac{d^{m_{1}-n-2}}{d w^{m_{1}-n-2}}\left[\frac{\left(w-z_{2}\right)^{n} g_{1}(w)}{\left(m_{1}-n-2\right) !}\right]\right|_{w=z_{1}}+\left.\frac{d^{m_{2}-n-1}}{d w^{m_{2}-n-1}}\left[\frac{\left(w-z_{1}\right)^{n+1} g_{2}(w)}{\left(m_{2}-n-1\right) !}\right]\right|_{w=z_{2}}\right\}
\end{aligned}
$$

From here, equations (18) and (19) follow after straightforward computations.

Remark 2. Let $z$ be a real or complex variable and $z_{1}, z_{2}\left(z_{1} \neq z_{2}\right)$ two real or complex numbers. Suppose that $g_{1}(z) \equiv\left(z-z_{1}\right)^{m_{1}} f(z)$ is $n$-times differentiable at $z_{1}$ and $g_{2}(z) \equiv\left(z-z_{2}\right)^{m_{2}} f(z)$ is $n$-times differentiable at $z_{2}$. Define

$$
g(z) \equiv f(z)-\sum_{n=0}^{M-1}\left[c_{n}\left(z_{1}, z_{2}\right)\left(z-z_{1}\right)+c_{n}\left(z_{2}, z_{1}\right)\left(z-z_{2}\right)\right]\left(z-z_{1}\right)^{-n-1}\left(z-z_{2}\right)^{-n-1}
$$

where $M \equiv \operatorname{Max}\left\{m_{1}, m_{2}\right\}$. Then, the thesis of Proposition 2 holds for $f(z)$ replaced by $g(z)$. Moreover, if $\left(z-z_{1}\right)^{m_{1}}\left(z-z_{2}\right)^{m_{2}} f(z)$ is an analytic function in $\Omega$, then the thesis of Theorem 1 applies to $g(z)$.

\section{Two-point Taylor-Laurent expansions}

Theorem 3. Let $\Omega_{0}$ and $\Omega$ be closed and open sets, respectively, of the complex plane, and $\Omega_{0} \subset \Omega \subset \mathbb{C}$. Let $f(z)$ be an analytic function on $\Omega \backslash \Omega_{0}, z_{1} \in \Omega_{0}$ and $z_{2} \in \Omega \backslash \Omega_{0}$. Then, for $z \in \Omega \backslash \Omega_{0}, f(z)$ admits the Taylor-Laurent expansion

$$
\begin{aligned}
f(z)= & \sum_{n=0}^{N-1}\left[d_{n}\left(z_{1}, z_{2}\right)\left(z-z_{1}\right)+d_{n}\left(z_{2}, z_{1}\right)\left(z-z_{2}\right)\right]\left(z-z_{1}\right)^{n}\left(z-z_{2}\right)^{n}+ \\
& \sum_{n=0}^{N-1} e_{n}\left(z_{1}, z_{2}\right)\left(z-z_{2}\right)^{n}\left(z-z_{1}\right)^{-n-1}+r_{N}\left(z_{1}, z_{2} ; z\right),
\end{aligned}
$$


where the coefficients $d_{n}\left(z_{1}, z_{2}\right), d_{n}\left(z_{2}, z_{1}\right)$ and $e_{n}\left(z_{1}, z_{2}\right)$ of the expansion are given by the Cauchy integrals

$$
d_{n}\left(z_{1}, z_{2}\right) \equiv \frac{1}{2 \pi i\left(z_{2}-z_{1}\right)} \int_{\Gamma_{1}} \frac{f(w) d w}{\left(w-z_{1}\right)^{n}\left(w-z_{2}\right)^{n+1}}
$$

and

$$
e_{n}\left(z_{1}, z_{2}\right) \equiv \frac{z_{1}-z_{2}}{2 \pi i} \int_{\Gamma_{2}} \frac{\left(w-z_{1}\right)^{n}}{\left(w-z_{2}\right)^{n+1}} f(w) d w
$$

The remainder term $r_{N}\left(z_{1}, z_{2} ; z\right)$ is given by the Cauchy integrals

$$
\begin{aligned}
r_{N}\left(z_{1}, z_{2} ; z\right) \equiv & \frac{1}{2 \pi i} \int_{\Gamma_{1}} \frac{f(w) d w}{\left(w-z_{1}\right)^{N}\left(w-z_{2}\right)^{N}(w-z)}\left(z-z_{1}\right)^{N}\left(z-z_{2}\right)^{N}- \\
& \frac{1}{2 \pi i} \int_{\Gamma_{2}} \frac{\left(w-z_{1}\right)^{N} f(w) d w}{\left(w-z_{2}\right)^{N}(w-z)} \frac{\left(z-z_{2}\right)^{N}}{\left(z-z_{1}\right)^{N}} .
\end{aligned}
$$

In these integrals, the contours of integration $\Gamma_{1}$ and $\Gamma_{2}$ are simple closed loops contained in $\Omega \backslash \Omega_{0}$ which encircle $\Omega_{0}$ in the counterclockwise direction. Moreover, $\Gamma_{2}$ does not contain the points $z$ and $z_{2}$ inside, whereas $\Gamma_{1}$ encircles $\Gamma_{2}$ and the points $z$ and $z_{2}$ (see Figure $6(a)$ ).

The expansion (20) is convergent in the region (Figure 7)

$$
D_{z_{1}, z_{2}} \equiv\left\{z \in \Omega \backslash \Omega_{0}, \quad\left|\left(z-z_{1}\right)\left(z-z_{2}\right)\right|<r_{1} \text { and }\left|z-z_{2}\right|<r_{2}\left|z-z_{1}\right|\right\}
$$

where $r_{1} \equiv \operatorname{Inf}_{w \in \mathbb{C} \backslash \Omega}\left\{\left|\left(w-z_{1}\right)\left(w-z_{2}\right)\right|\right\}$ and $r_{2} \equiv \operatorname{Inf}_{w \in \Omega_{0}}\left\{\left|\left(w-z_{2}\right)\left(w-z_{1}\right)^{-1}\right|\right\}$.

Proof. By Cauchy's theorem,

$$
f(z)=\frac{1}{2 \pi i} \int_{\Gamma_{1}} \frac{f(w) d w}{w-z}-\frac{1}{2 \pi i} \int_{\Gamma_{2}} \frac{f(w) d w}{w-z},
$$

where $\Gamma_{1}$ and $\Gamma_{2}$ are the contours defined above. We substitute (7)-(8) into the first integral above and

$$
\frac{1}{w-z}=\frac{z_{2}-z_{1}}{\left(z-z_{1}\right)\left(w-z_{2}\right)} \frac{1}{1-u}, \quad u \equiv \frac{\left(w-z_{1}\right)\left(z-z_{2}\right)}{\left(z-z_{1}\right)\left(w-z_{2}\right)}
$$

into the second one. Now we introduce the expansion (9) of the factor $(1-u)^{-1}$ in both integrals in (25). After straightforward calculations we obtain (20)-(23).

For any $z$ verifying (24), we can take simple closed loops $\Gamma_{1}$ and $\Gamma_{2}$ in $\Omega \backslash \Omega_{0}$ such that $\left|\left(z-z_{1}\right)\left(z-z_{2}\right)\right|<\left|\left(w-z_{1}\right)\left(w-z_{2}\right)\right| \forall w \in \Gamma_{1}$ and $\left|\left(z-z_{1}\right)\left(w-z_{2}\right)\right|>\left|\left(w-z_{1}\right)\left(z-z_{2}\right)\right|$ $\forall w \in \Gamma_{2}$ (see Figure 6 (b)). On these contours $|f(w)|$ is bounded by some constant $C:|f(w)| \leq C$. Introducing these bounds in (23) we see that $\lim _{N \rightarrow \infty} r_{N}\left(z_{1}, z_{2} ; z\right)=0$ and the proof follows. 


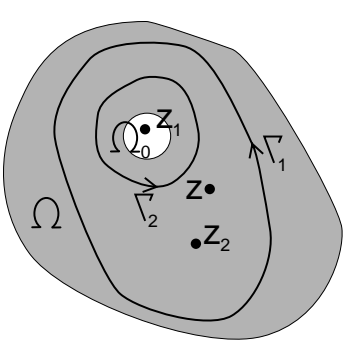

(a)

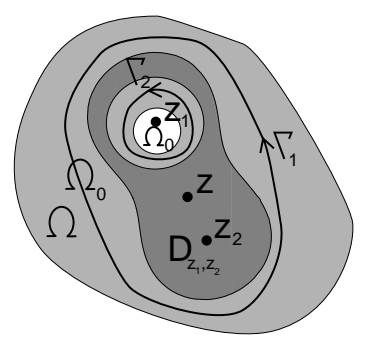

(b)

Figure 6. (a) Contours $\Gamma_{1}$ and $\Gamma_{2}$ in the integrals (20)-(23). (b) For $z \in D_{z_{1}, z_{2}}$, we can take a contour $\Gamma_{2}$ situated between $\Omega_{0}$ and $D_{z_{1}, z_{2}}$ and a contour $\Gamma_{1}$ in $\Omega$ which contains $D_{z_{1}, z_{2}}$ inside. Therefore, $\left|\left(z-z_{1}\right)\left(z-z_{2}\right)\right|<\left|\left(w-z_{1}\right)\left(w-z_{2}\right)\right| \forall w \in \Gamma_{1}$ and $\left|\left(w-z_{1}\right)\left(z-z_{2}\right)\right|<$ $\left|\left(z-z_{1}\right)\left(w-z_{2}\right)\right| \forall w \in \Gamma_{2}$.

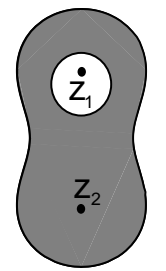

(a) $4 r_{1}>\left|z_{1}-z_{2}\right|^{2}, r_{2}>1$

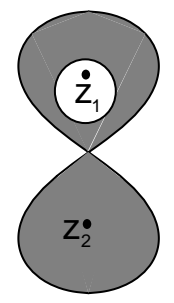

(d) $4 r_{1}=\left|z_{1}-z_{2}\right|^{2}, r_{2}>1$

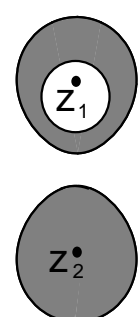

(g) $4 r_{1}<\left|z_{1}-z_{2}\right|^{2}, r_{2}>1$

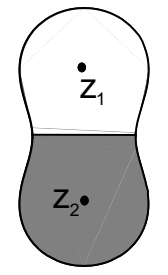

(b) $4 r_{1}>\left|z_{1}-z_{2}\right|^{2}, r_{2}=1$

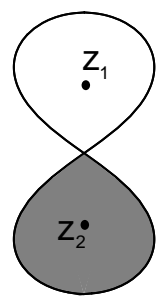

(e) $4 r_{1}=\left|z_{1}-z_{2}\right|^{2}, r_{2}=1$

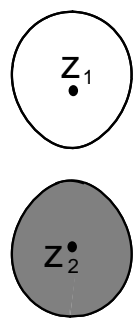

(h) $4 r_{1}<\left|z_{1}-z_{2}\right|^{2}, r_{2}=1$

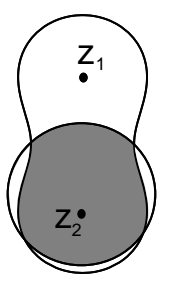

(c) $4 r_{1}>\left|z_{1}-z_{2}\right|^{2}, r_{2}<1$

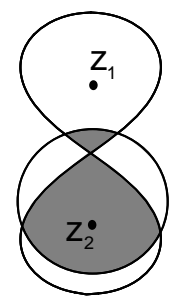

(f) $4 r_{1}=\left|z_{1}-z_{2}\right|^{2}, r_{2}<1$

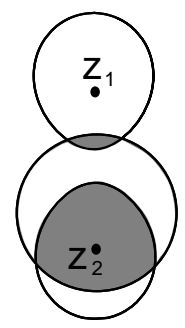

Figure 7. The region $D_{z_{1}, z_{2}}$ defined in Theorem 3 is given by $D_{z_{1}, z_{2}}=D_{1} \cap D_{2}$, where $D_{1}$ is the Cassini oval of focus $z_{1}$ and $z_{2}$ and parameter $r_{1}$. On the other hand, for $r_{2}<1\left(r_{2}>1\right), D_{2}$ is the interior (exterior) of the circle of center $z_{1}+\left(1-r_{2}^{2}\right)^{-1}\left(z_{2}-z_{1}\right)=z_{2}+r_{2}^{2}\left(r_{2}^{2}-1\right)^{-1}\left(z_{1}-z_{2}\right)$ and radius $\left|z_{1}-z_{2}\right| r_{2} /\left|r_{2}^{2}-1\right|$. For $r_{2}=1, D_{2}$ is just the half plane $\left|z-z_{2}\right|<\left|z-z_{1}\right|$. The 
shape of the Cassini annulus depends on the relative size of the parameters $\sqrt{r_{1}}, \sqrt{r_{2}}$ and the focal distance $\left|z_{1}-z_{2}\right|$.

If the only singularities of $f(z)$ inside $\Omega_{0}$ are just poles at $z_{1}$, then an alternative formula to (21)-(22) to compute the coefficients of the above two-point Taylor-Laurent expansion is given in the following proposition.

Proposition 4. Suppose that $g(z) \equiv\left(z-z_{1}\right)^{m} f(z)$ is an analytic function in $\Omega$ for certain $m \in \mathbb{N}$. Then, coefficients $d_{n}\left(z_{1}, z_{2}\right)$ and $d_{n}\left(z_{2}, z_{1}\right)$ in the expansion (20) are also given by the formulas:

$$
\begin{gathered}
d_{0}\left(z_{1}, z_{2}\right)=\frac{f\left(z_{2}\right)}{z_{2}-z_{1}}-\sum_{k=0}^{m-1} \frac{1}{(m-k-1) !} \frac{g^{(m-k-1)}\left(z_{1}\right)}{\left(z_{2}-z_{1}\right)^{k+2}}, \\
d_{0}\left(z_{2}, z_{1}\right)=\frac{1}{m !} \frac{g^{(m)}\left(z_{1}\right)}{z_{1}-z_{2}},
\end{gathered}
$$

and, for $n=1,2,3 \ldots$,

$$
\begin{aligned}
d_{n}\left(z_{1}, z_{2}\right)= & -\frac{(-1)^{n}}{n !}\left\{\sum_{k=0}^{m+n-1} \frac{(n+k) !}{k !(m+n-k-1) !} \frac{g^{(m+n-k-1)}\left(z_{1}\right)}{\left(z_{2}-z_{1}\right)^{n+k+2}}+\right. \\
n \sum_{k=0}^{n} \frac{(n+k-1) !}{k !(n-k) !} \frac{f^{(n-k)}\left(z_{2}\right)}{\left.\left(z_{1}-z_{2}\right)^{n+k+1}\right\}} & \\
d_{n}\left(z_{2}, z_{1}\right)= & -\frac{(-1)^{n}}{n !}\left\{n \sum_{k=0}^{m+n} \frac{(n+k-1) !}{k !(m+n-k) !} \frac{g^{(m+n-k)}\left(z_{1}\right)}{\left(z_{2}-z_{1}\right)^{n+k+1}}+\right. \\
& \left.\sum_{k=0}^{n-1} \frac{(n+k) !}{k !(n-k-1) !} \frac{f^{(n-k-1)}\left(z_{2}\right)}{\left(z_{1}-z_{2}\right)^{n+k+2}}\right\} .
\end{aligned}
$$

For $n=0,1,2, \ldots$, coefficients $e_{n}\left(z_{1}, z_{2}\right)$ are given by

$$
e_{n}\left(z_{1}, z_{2}\right)=\frac{(-1)^{n}}{n !} \sum_{k=0}^{m-n-1} \frac{(n+k) !}{k !(m-n-k-1) !} \frac{g^{(m-n-k-1)}\left(z_{1}\right)}{\left(z_{2}-z_{1}\right)^{n+k}}
$$

Proof. We deform both, the contour $\Gamma_{1}$ in equation (21) and the contour $\Gamma_{2}$ in equation (22) to any contour of the form $\mathcal{C}_{1} \cup \mathcal{C}_{2}$ contained in $\Omega$, where $\mathcal{C}_{1}\left(\mathcal{C}_{2}\right)$ is a simple closed loop which encircles the point $z_{1}\left(z_{2}\right)$ in the counterclockwise direction and does not 
contain the point $z_{2}\left(z_{1}\right)$ inside (see Figure $3(\mathrm{c})$ ). Then,

$$
\begin{aligned}
d_{n}\left(z_{1}, z_{2}\right)= & \frac{1}{2 \pi i\left(z_{2}-z_{1}\right)}\left\{\int_{\mathcal{C}_{1}} \frac{g(w)}{\left(w-z_{2}\right)^{n+1}} \frac{d w}{\left(w-z_{1}\right)^{n+m}}+\right. \\
& \left.\int_{\mathcal{C}_{2}} \frac{f(w)}{\left(w-z_{1}\right)^{n}} \frac{d w}{\left(w-z_{2}\right)^{n+1}}\right\}= \\
& \frac{1}{\left(z_{2}-z_{1}\right)}\left\{\left.\frac{1}{(n+m-1) !} \frac{d^{n+m-1}}{d w^{n+m-1}} \frac{g_{1}(w)}{\left(w-z_{2}\right)^{n+1}}\right|_{w=z_{1}}+\right. \\
& \left.\left.\frac{1}{n !} \frac{d^{n}}{d w^{n}} \frac{f(w)}{\left(w-z_{1}\right)^{n}}\right|_{w=z_{2}}\right\},
\end{aligned}
$$

an analog formula for $d_{n}\left(z_{2}, z_{1}\right)$, and

$$
\begin{aligned}
e_{n}\left(z_{1}, z_{2}\right)= & \frac{z_{1}-z_{2}}{2 \pi i} \int_{\mathcal{C}_{1}} \frac{g(w)}{\left(w-z_{2}\right)^{n+1}} \frac{d w}{\left(w-z_{1}\right)^{m-n}}= \\
& \left.\left(z_{1}-z_{2}\right) \frac{1}{(m-n-1) !} \frac{d^{m-n-1}}{d w^{m-n-1}} \frac{g(w)}{\left(w-z_{2}\right)^{n+1}}\right|_{w=z_{1}} .
\end{aligned}
$$

From here, equations (27)-(30) follow after straightforward computations.

Remark 3. Let $z$ be a real or complex variable and $z_{1}$ and $z_{2}\left(z_{1} \neq z_{2}\right)$ two real or complex numbers. Suppose that $\left(z-z_{1}\right)^{m} f(z)$ is $n$-times differentiable at $z_{1}$ for certain $m \in \mathbb{N}$ and $f(z)$ is $n$-times differentiable at $z_{2}$. Define

$$
g(z) \equiv f(z)-\sum_{n=0}^{m-1} e_{n}\left(z_{1}, z_{2}\right)\left(z-z_{1}\right)^{-n-1}\left(z-z_{2}\right)^{n} .
$$

Then, the thesis of Proposition 2 holds for $g(z)$. If moreover, $\left(z-z_{1}\right)^{m} f(z)$ is an analytic function in $\Omega$, then the thesis of Theorem 1 applies to $g(z)$.

\section{Acknowledgements}

J. L. López wants to thank the C.W.I. of Amsterdam for its scientific and financial support during the realization of this work. The financial support of the saving bank Caja Rural de Navarra is also acknowledged. 


\section{References}

[1] C. Chester, B. Friedman, and F. Ursell, An extension of the method of steepest descent, Proc. Cambridge Philos. Soc., 53 (1957) 599-611.

[2] José L. Lopez And Nico M. Temme, Asymptotic expansions of Charlier, Laguerre and Jacobi polynomials. Submited, 2002.

[3] José L. Lopez and Nico M. Temme, Two-point Taylor expansions of analytic functions. Submited, 2002.

[4] Raimundas Vidunas and Nico M. Temme, Symbolic evaluation of coefficients in Airy-type asymptotic expansions. CWI Report MAS-R0118. Accepted for publication in Journal of Mathematical Analysis and Applications (2001).

[5] J. L. WALSH, Interpolation and Approximation by rational functions in the complex domain, American Mathematical Society, Providence, 1969.

[6] E.T. Whittaker and G.N. Watson, A course of modern analysis, Cambridge University Press, London and New York, 1927.

[7] R. Wong, Asymptotic Approximations of Integrals, Academic Press, New York, 1989. 